\title{
EL TALLER EDUCATIVO: SISTEMATIZACIÓN DE UN MODELO DE FORMACIÓN DE PSICÓLOGOS/AS, EN DOS UNIVERSIDADES DE LA QUINTA REGIÓN
}

\author{
Claudia Carrasco ${ }^{1}$, María Julia Baltar ${ }^{1}$, Mabel Bórquez ${ }^{2}$, \\ Claudia Cuneo $^{1}$, Cecilia De la Cerda ${ }^{3}$
}

\begin{abstract}
RESUMEN
Este artículo sistematiza un Modelo de Formación de Psicólogos/as en el área de Psicología Educacional, en dos universidades de la V Región, que busca promover la autonomía y la participación, basado en los Talleres de Educación Democrática y la Interacción Centrada en un Tema. Se llevan a cabo tres estudios cualitativos entre los años 2005 y 2010 con el fin de conocer los principales aprendizajes identitarios que significan los estudiantes formados bajo este modelo, quienes señalan que la modalidad de trabajo potencia su capacidad de cuestionar críticamente su entorno, valorando los pequeños cambios como promotores de cambios mayores. Estos aprendizajes construirían un Psicólogo/a conciente de las implicancias éticas de su rol, llevando estos aprendizajes a otros campos de acción.
\end{abstract}

Palabras clave: Talleres Educativos, Formación de Psicólogos/as, Implicancias profesionales

\section{THE EDUCATIVE WORKSHOP: SYSTEMATIZING OF AN EDUCATION MODEL FOR PSYCHOLOGISTS IN TWO UNIVERSITIES IN THE FIFTH REGION}

\section{ABSTRACT}

This article systematizes an Education Model for Psychologists in the area of Educational Psychology in two universities of the Fifth Region in Chile, seeking to promote autonomy and participation based on the Workshops of Democratic Education and the Interaction Focused in a Topic. Three qualitative researches have been carried out between 2005 and 2010 in order to know the principal lessons generated by professional identity and taken by students formed under this model, who claim that this modality of work promotes their aptitudes to critically question the environment valuing the small changes as promoters of major changes. This learning would construct a psychologist aware of all the ethical implications of hisher role, taking this learning into other fields of action.

Key words: Educational Workshops, Formation of Psychologists, Professional implications

1 Escuela de Psicología, Pontificia Universidad Católica de Valparaíso. Contacto: nadieseeducasolo@gmail.com

2 Escuela de Psicología, Universidad Internacional SEK.

3 Unidad de Psicoterapia Dinámica, Instituto Psiquiátrico Dr. José H. Barak. 


\section{EL TALLER EDUCATIVO: SISTEMATIZACIÓN DE UN MODELO DE FORMACIÓN DE PSICÓLOGOS/AS, EN DOS UNIVERSIDADES DE LA QUINTA REGIÓN}

\section{Antecedentes y contexto}

Para Bernasconi y Rojas (2003), desde la creación en 1842 de la Universidad de Chile la historia de la educación superior estuvo a cargo del Estado. En la práctica, esto significó apoyo financiero por parte del Estado a las universidades privadas establecidas desde la fundación de la Universidad Católica de Chile en 1888 e implicó, a la vez, que los estudiantes de estas instituciones tuvieron que rendir exámenes finales ante la Universidad de Chile hasta mediados de 1950. Entre 1919 y 1956 se fundaron la Universidad de Concepción, la Universidad Técnica Federico Santa María, la Universidad Católica de Valparaíso, la Universidad Austral de Chile y la Universidad del Norte (que después se convertiría en la Universidad Católica del Norte).

De casi 20.000 matrículas de pregrado en 1957, el sistema educativo universitario chileno aumentó en 10 años a más de 55.000 alumnos, lo cual representaba una tasa bruta de matrícula del $7 \%$ para la cohorte de 20 a 24 años de edad en 1967 (Brunner 1986, citado en Bernasconi y Rojas, 2003). Ese mismo año se inicia en Chile la Reforma Universitaria, la que es descrita por Bernasconi y Rojas (2003) como un proceso de intensa discusión acerca de la naturaleza de la universidad y su papel en la sociedad chilena, lo que para Del Mazo (1966) constituye parte del proceso de reformas latinoamericanas en educación superior (Bernasconi y Rojas, 2003).

En esta vorágine de transformaciones, la creación del primer centro de formación de Psicología en Chile tiene su hito fundacional en la carrera de Psicología en la Universidad de Chile en 1946, pionera también en América Latina. El segundo centro de formación fue creado en 1957, en la Pontificia Universidad Católica de Chile, permaneciendo como los únicos existentes en el país hasta el año 1982 
(Villegas, 1998; Descouvriers, 1999). En 1974 se realiza en Bogotá la Primera Conferencia Latinoamericana sobre de Entrenamiento en Psicología, en la cual se presenta el modelo latinoamericano de formación en psicología denominado Modelo Bogotá, que sienta las bases para la formación de pregrado, las cuales son adoptadas completamente por las universidades chilenas (Vilanova, 2003).

Posteriormente, gracias a la Ley General de Universidades de 1981 se aumentan los centros educativos que dictan la carrera de Psicología (Descouvriers, 1999). Sin embargo, solo unos años después de la instauración del MERCOSUR ${ }^{4}$ se renovó el interés de la Psicología en la formación de pregrado de esta carrera, formando en 1994 el Comité Coordinador de psicólogos/as del MERCOSUR, estableciendo así los principios para la formación de psicólogos/as (Vilanova, 2003).

Esto marca un hito a partir del cual comienzan a establecerse acuerdos globales, los cuales dan como resultado, entre otros, la realización del II Encuentro de Entidades de Psicología de América Latina en Santiago de Chile el 2001, donde se plantea que el psicólogo/a debería planificar, desarrollar, evaluar y diagnosticar procesos y programas en las distintas áreas de la psicología, además de definir, aplicar y evaluar estrategias de intervención para atender necesidades preventivas, terapéuticas y de desarrollo de grupos y organizaciones (Comité coordinador de psicólogos del Mercosur, 2001).

Atendiendo a Avendaño (1996), los planes de estudio de Psicología en Chile se caracterizarían desde entonces por un currículo nuclear, con predominio de objetivos cognitivos y baja formación en el ámbito ético. Según Bernasconi y Rojas (2003) esto se debería, fundamentalmente, a la orientación de la educación superior de la época hasta el día de hoy, ya que para estos autores el énfasis de las políticas de educación superior de la década de los noventa y principios de la década del 2000 estuvo dado, principalmente, por los ejes de la calidad y la equidad y, en forma secundaria, por el objetivo de vincular más estrechamente a la educación superior con el desarrollo nacional y regional, por lo que el bien común nunca

4 Mercado Común del Sur, integrado por Argentina, Brasil, Paraguay, Uruguay y Venezuela. 
132 EL TALLER EDUCATIVO: SISTEMATIZACIÓN DE UN MODELO DE FORMACIÓN DE PSICÓLOGOS/AS, EN DOS UNIVERSIDADES DE LA QUINTA REGIÓN - M. J. Baltar, C. Carrasco, M. Bórquez, C. Cuneo, C. De la Cerda

volvió ser el eje articulador de las universidades como lo fue en la década de los sesenta.

Para Winkler, Pasmanik, Alvear y Reyes (2007) la ética, entendida como una orientación hacia el bien común, debe ser una consideración imprescindible del ejercicio de la Psicología como disciplina, lo que sería difícil de cautelar, ya que no existe al respecto obligatoriedad de colegiatura en los colegios profesionales, los que además no poseen la tuición ética.

Ahora bien, entre el año 1996 y el año 2000 aumentó en un $25,8 \%$ la cantidad de planteles universitarios que impartía la carrera de Psicología, siendo más evidente este incremento para las instituciones privadas con un 19,3\% (Winkler y otros, 2007). Al año 2004, existían 39 universidades que dictaban un total de 95 programas de pregrado en Psicología (Consejo Superior de Educación, 2004, citado en Makrinov, Scharager y Molina, 2005). Para el año 2005 existían en el país un total de 40 casas de estudio con la carrera de Psicología (Winkler y otros, 2007), llegando al año 2011 a un total de 129 programas (Consejo Nacional de Educación, 2011).

Para Winkler y otros (2007), la distribución geográfica de los programas de Psicología en Chile se concentra en la zona central, especialmente en Santiago, Viña del Mar y Valparaíso, aunque en la Quinta Región el ingreso medio (478.023 pesos) es el más bajo de todo Chile (Meller, Lara y Valdés, 2009b).

Por su lado, en una preocupación por conocer el ámbito de desempeño de la creciente cantidad de psicólogos en Chile, Makrinov y otros (2005) realizaron un estudio orientado a dar cuenta de distintos aspectos del campo y del rol del psicólogo, cuyos resultados analizan en relación con los aportes de Morales, Díaz, Scharager y Sziklai (1988) y concluyen que habría disminuido la cantidad de psicólogos que ejercen en el ámbito clínico, aumentando los que trabajan en el área organizacional y los que se dedican a la docencia, manteniéndose la cantidad que se desempeña en el ámbito educacional. De todas maneras, los indicadores muestran que la psicología clínica sigue siendo el área más demandada, con el 58,3\% de los psicólogos/as, 
quedando el $22,9 \%$ en la especialidad organizacional y el 9,4\%, en la educacional (Makrinov y otros, 2005).

A la luz de todos estos resultados, la red que convoca a las Escuelas de Psicología de las Universidades de Chile, La Frontera, La Serena, Valparaíso, Talca, Tarapacá y de Santiago de Chile, desarrolla en el periodo 2006-2011 el proyecto MECESUP denominado "Mejoramiento de la formación general y específica de los profesionales psicólogos del Consorcio de Universidades del Estado mediante el diseño e implementación de un marco curricular común basado en competencias", con la finalidad de crear un perfil de egreso común entre los estudiantes de estas siete universidades.

Por su lado, Linn (2007) publicó los resultados de una investigación encargada por el Colegio de Psicólogos de Chile que concluye que, aunque las preferencias seguían manteniendo el mismo orden del 2005, los porcentajes habrían variado, incorporándose áreas emergentes de la Psicología. De este modo, las especializaciones de psicólogos/as se distribuían de la siguiente forma: un 40,7\% en el área clínica, 19\% en laboral, 10,2\% en educacional, 9,3\% en socialcomunitaria, $7,9 \%$ en docencia, $4,7 \%$ en jurídica y $2,4 \%$ en salud y rehabilitación. El área educacional se encuentra en la tercera posición de opciones de especialidad, pero si se trata de la percepción de satisfacción, esta área desplaza a la psicología clínica con un 35,3\% de profesionales totalmente satisfechos en su especialidad, frente a un $31 \%$ en el área clínica.

En el ámbito gremial, en los años cincuenta se crea la Asociación de Psicólogos de Chile, y posteriormente en 1968 se funda el Colegio de Psicólogos de Chile, que cuenta al año 2011 con más de 2.300 afiliados. Debido a la necesaria diferenciación surgida del ámbito de trabajo, al requerimiento por mejorar la calidad de los servicios y a la búsqueda de mecanismos de especialización, surgieron en Chile sociedades y asociaciones de psicología. Si bien la única área de especialización de la psicología acreditada en Chile a la fecha es la de psicoterapia en el área clínica (Urrutia, 2002), el año 2008 y con el apoyo del Colegio de Psicólogos, se reactiva la creación de la Asociación Nacional de Psicólogos Educacionales (ANPSE). 
Actualmente, la ANPSE busca desarrollar acciones vinculadas con el desarrollo de la ética y los requerimientos en torno a la calidad en la formación y desempeño de los profesionales, entre otros temas. Estas acciones llevaron a visibilizar el rol de la psicología educacional frente al Ministerio de Educación, a la Comisión de Formación Inicial Docente del Centro de Perfeccionamiento Educativo e Investigación Pedagógico (CPEIP) y a la Comisión de Educación de la Cámara de Diputados (ANPSE, 2011). Este escenario vuelve interesante la formación de pregrado de psicólogos/as en el área educacional.

La necesidad de innovar e investigar la docencia que se estaba construyendo emerge en las autoras de este artículo durante el transcurso de las prácticas pedagógicas de enseñanza de la psicología aplicada al área educacional en dos universidades de la Quinta Región de Chile. Desde la perspectiva del currículo fue necesario analizar, críticamente, aquellos aspectos y momentos que estarían dando cuenta de los efectos reproductivos de la cultura dominante, por lo cual se requería revisar el fenómeno en dos niveles: desde lo manifiesto y declarado (currículo explícito), esto es, los objetivos, contenidos, metodologías de enseñanza y evaluación, como también a nivel de lo implícito (currículo oculto), es decir, poniendo un especial y permanente énfasis y atención a lo que inadvertidamente podría estarse enseñando y/o aprendiendo. De esta manera, se ha ido construyendo a través de 10 años de ejercicio docente, en conjunto con un grupo de colaboradores (ex alumnos, ayudantes y profesores), una propuesta de enseñanza y aprendizaje de la psicología educacional que considera diversos elementos teóricos y prácticos que intentan producir una cultura crítica en las relaciones entre la psicología y la sociedad. En esta propuesta en desarrollo, destacan los siguientes aspectos:

- Prácticas de enseñanza y de aprendizaje a través de la temprana inserción supervisada de nuestros estudiantes en escuelas y del acompañamiento de los procesos en actividades de clases a través de metodologías de talleres educativos.

- Potenciación de relaciones sociales democráticas, tanto en el aula como en los trabajos de terreno, a nivel de la enseñanza, aprendizaje y evaluación.

- Permanente análisis en torno a las implicancias individuales, 
institucionales y sociales que están en la base de los discursos y de las prácticas implementadas.

Así, esta propuesta se encuentra fundamentada, por una parte, en los enfoques psicológicos humanistas y, por otra parte, en la tradición del constructivismo y de la pedagogía crítica. En términos disciplinarios, las cátedras de psicología educacional en ambas universidades se basan en un cuestionamiento de las dimensiones éticas y políticas de la psicología (Montero, 2001), así como en un permanente cuestionamiento respecto de la posición del psicólogo y su vinculación con el poder (Wiesenfeld, 2000).

En la malla curricular de ambas universidades, el ramo de psicología educacional aparece en el $5^{\circ}$ semestre, continuando durante el $7^{\circ}$ semestre, cuando finaliza en una y continúa hasta $9^{\circ}$ y $10^{\circ}$ semestre en la otra. En el marco de esta propuesta, los estudiantes deben llevar a cabo un estudio de la realidad educativa, implementando acciones preventivas primarias o transformadoras de rasgos de la cultura escolar (Coll, Marchesi y Palacios, 1990). En la universidad 1 (tradicional privada) se trabaja en la modalidad de taller educativo con mayor énfasis durante el $5^{\circ}$ año de carrera, mientras que en la universidad 2 (privada) se trabaja usando esta modalidad con mayor énfasis en el $3^{\text {er }}$ año. Esto se vincula con el hecho de que los contenidos curriculares en ambas universidades coinciden en estos cursos, esperándose de los estudiantes intervenciones colectivas dirigidas a la mejora escolar en los establecimientos en los que deben realizar el trabajo teórico y práctico. De este modo, si bien las actividades de planificación de ambas cátedras se asemejan bastante en ambas instituciones (ya que se trabaja en la docencia universitaria en ambas realidades de forma paralela), las adecuaciones del diseño curricular en ellas se relacionan con los momentos formativos de cada universidad. En este sentido, ya que en la universidad 1 los estudiantes han atravesado dos semestres de formación en el área, se espera un mayor dominio conceptual y de competencias profesionales que en los estudiantes de la universidad 2, quienes se encuentran por primera vez con el área educacional, llevando tan solo dos años previamente en el pregrado. 

PSICÓLOGOS/AS, EN DOS UNIVERSIDADES DE LA QUINTA REGIÓN - M. J. Baltar, C. Carrasco, M. Bórquez, C. Cuneo, C. De la Cerda

Sin embargo, en ambos casos la evaluación es concebida como parte del proceso de enseñanza y aprendizaje y los procedimientos evaluativos se encuentran focalizados en el análisis crítico de los trabajos en terreno respecto de los aprendizajes construidos a partir de ellos, destacando la construcción de "diarios de aprendizaje", los que al finalizar el semestre (y de forma parcial durante el mismo) deben ser analizados por sus autores, realizando así un análisis de los análisis, lo que se construye bajo la denominación de "metaanálisis" a través de un informe escrito elaborado por cada estudiante.

Este modelo de formación de los talleres educativos tiene influencias de los talleres de educación democrática (TED) (Cerda, Aránguiz, Cid, y Miranda, 1994), con elementos del enfoque de interacción centrada en un tema (ICT) (Cohn, 1991, citado en Hernández Arista, 2000) y su planificación considera tres momentos: actividad inicial de rompehielos o caldeamiento, actividad central de discusión en pequeños grupos y plenario y, por último, evaluación de la sesión, lo que se sintetiza en la siguiente tabla:

Tabla $n^{\circ}$ 1. Fases de la planificación del Taller Educativo

\begin{tabular}{|l|l|l|}
\hline $\begin{array}{l}\text { PRIMERA } \\
\text { ACTIVIDAD }\end{array}$ & $\begin{array}{l}\text { Rompehielos } \\
\text { o } \\
\text { caldeamiento }\end{array}$ & $\begin{array}{l}\text { Actividad de recreación de experiencias y conocimientos previos } \\
\text { en relación con los contenidos conceptuales, procedimentales y/o } \\
\text { estratégicos, a través de una amplia variedad de dinámicas grupales } \\
\text { que son reflexionadas posteriormente en el plenario. }\end{array}$ \\
\hline $\begin{array}{l}\text { SEGUNDA } \\
\text { ACTIVIDAD }\end{array}$ & $\begin{array}{l}\text { Actividad } \\
\text { central }\end{array}$ & $\begin{array}{l}\text { Actividad teórico-práctica con apoyo bibliográfico de lectura previa o } \\
\text { presencial, combinada con discusión en pequeños grupos y posterior } \\
\text { plenario, a través de preguntas guías que orienten la reflexión, } \\
\text { contextualización y construcción de relaciones pertinentes, relevantes } \\
\text { y significativas con la experiencia de los trabajos de terreno. } \\
\text { Se utilizan también técnicas proyectivas a través de actividades tales } \\
\text { como collage, esculturas humanas y en greda o plastilina, pintura, } \\
\text { dibujo, juegos de rol, entre otras, cuya objetivación de significados y } \\
\text { sentidos se realiza en plenario. } \\
\text { También se realizan actividades en grupos de amigos críticos, } \\
\text { compuestos por 2 o 3 grupos de trabajo de campo en las que se } \\
\text { comparten las vivencias en terreno, se contienen, apoyan y asesoran } \\
\text { mutuamente. } \\
\text { Eventualmente se llevan a cabo presentaciones al grupo-curso, tanto de } \\
\text { las experiencias para asesoría colectiva como de elaboraciones teóricas } \\
\text { que sean pertinentes y oportunas. }\end{array}$ \\
\hline $\begin{array}{l}\text { TERCERA } \\
\text { ACTIVIDAD }\end{array}$ & $\begin{array}{l}\text { Evaluación de } \\
\text { la clase }\end{array}$ & $\begin{array}{l}\text { Etapa en la que los psicólogos en formación evalúan los aprendizajes } \\
\text { e interacciones construidas a partir de alguna técnica de características } \\
\text { proyectivas. }\end{array}$ \\
\hline
\end{tabular}


En este contexto de innovación curricular cabe preguntarse por los aprendizajes que construyen los estudiantes y las implicancias que estos conllevarían para retroalimentar la propuesta. El presente artículo busca determinar los aprendizajes profesionales más relevantes en este proceso de formación y analizar la evolución histórica de los mismos, derivados de entrevistas y de los informes de metaanálisis que construyen los estudiantes en relación con sus procesos de aprendizaje durante las diferentes experiencias de las cátedras a cargo del equipo que ha estado implementando estas innovaciones, con el fin de retroalimentar los objetivos y metodologías de esta experiencia formativa.

\section{Marco teórico de este estudio}

Para Baltar (2005) la educación sería a la vez individual y social, tanto en su realización como en los fines que persigue. Esta permanente discusión permearía cualquier intento de análisis del fenómeno educativo, por lo que los análisis que resultan pertinentes para la cultura escolar de establecimientos educativos escolares lo sería también para el contexto de la formación superior. En este sentido, López, Assael y Neumann (1991) se encargan de definir la cultura escolar como una red de significaciones que atribuyen los actores educativos a su realidad social, percibida por los agentes educativos de modo tal que la responsabilidad del fracaso escolar es atribuida a los estudiantes. De este modo, el fracaso es interpretado como una incapacidad de los alumnos para cumplir con los objetivos y, tal como analizan López, Carrasco, Morales, Ayala, López y Karmy (2011) al determinar donde reside el problema, la escuela externaliza a los alumnos fracasados. Esto tendría ciertas implicancias respecto de los procesos de transmisión de la cultura dominante y de la escuela como institución social encargada de ello.

Para Bourdieu y Passeron (2002) la realidad individual y social de la que nos habla Baltar (2005) es a la vez objetiva y subjetiva, a través de la relación entre el habitus y el campo de producción cultural. El habitus sería el producto de una incesante empresa de aprendizaje, inculcación, apropiación y control, el cual hace parte de todas las instituciones y campos sociales. Por su parte, los 
campos de producción cultural serían espacios sociales dinámicos y estructurados, en calidad de sistemas integrales de posiciones, donde los agentes sociales se relacionan de manera permanente y dinámica. Cada campo de producción cultural produciría una especie concreta de capital (cultural, social y económico) que, al adicionarse a otros capitales, conformaría el capital simbólico.

Atendiendo a estos planteamientos, el trabajo de adhesión al orden establecido se ejerce con la complicidad de los agentes que lo padecen, por lo que los dominados pliegan sus intereses, pensamientos y sentimientos a favor de los dominantes. Por esto, los actos de dominación presentan una doble dinámica: son actos de reconocimiento en la medida en que logran la aceptación de la legitimidad y del sentido impuestos por las relaciones de dominación y son también actos de desconocimiento, puesto que suponen la ignorancia del carácter arbitrario que los hace posibles. Para lograr estos efectos se requiere de un trabajo basado en acciones pedagógicas múltiples, capaces de sustituir la violencia física y psicológica en violencia simbólica, con la finalidad de inculcar el arbitrario cultural de clase o grupos sociales determinados en formas de conductas, saberes y disposiciones durables.

En este sentido, para las autoras toda acción pedagógica es objetivamente violencia simbólica y poder arbitrario, porque las relaciones que se dan entre los grupos que constituyen una sociedad originan el poder arbitrario que se da en la comunicación pedagógica. Además, los contenidos y significaciones que definen una cultura, en tanto sistema simbólico, no pueden deducirse en un principio universal, ni tampoco encuentran explicación en la naturaleza de las cosas. Para que la acción pedagógica sea eficaz, esta doble arbitrariedad debe mantenerse oculta, así como tampoco pueden aparecer completamente explicitados ni la manera arbitraria de la imposición, ni los contenidos que se inculcan.

Considerando estos planteamientos, lo que se transmite en la relación de aprendizaje no sería solo información, ya que es necesario el reconocimiento de la legitimidad de la autoridad pedagógica del profesor, que en cualquier circunstancia condiciona la recepción de la información convirtiéndola en formación. Para Bourdieu y Passeron 
(2002) la efectividad y productividad del trabajo pedagógico se podría evaluar por los siguientes factores: el grado de duración del habitus inculcado, la medida en que el habitus es transferible de una situación a otra y por la exhaustividad del habitus, es decir, por su capacidad para engendrar prácticas sociales que corresponden al orden cultural inculcado.

A pesar de los grandes aportes de esta teoría, Giroux (1983) cuestiona el hecho de que el pensamiento reflexivo y el agenciamiento histórico sean relegados a un detalle menos teórico. Para Giroux (1983) "el análisis de Bourdieu desarrolla poca fe en las clases y los grupos subordinados y poca esperanza en su habilidad o voluntad de reconstruir las condiciones bajo las cuales ellos viven, trabajan y aprenden" (p. 15).

Para este autor, Bourdieu y Passeron (2002) no son capaces de incluir en sus análisis el "agenciamiento humano" como potencial de transformación social. Esta concepción permite una visión dialéctica de la sociedad y posibilita la acción dirigida hacia la transformación de las condiciones de injusticia social. Esta visión fue trabajada en los planteamientos de Freire (2003), para quien el ser humano es un ser en construcción, es decir, inacabado. Esta incompletitud le permitiría construir y deconstruir su propia historia y, al mismo tiempo, recorrer caminos diferenciados en busca de su propio mejoramiento. Para el autor, esta incompletitud genera, además, el sentido de trascendencia, el cual consiste en adquirir conciencia de la finitud de sí mismo, hacia la trascendencia que promueve una unión que es de liberación. Entonces, en la concepción ontológica de Freire (2003), el ser humano poseería una postura dinámica en el mundo, formando parte de él a la vez que lo construiría.

\section{Metodología del estudio}

\subsection{Diseño}

Se llevaron a cabo tres estudios de corte transversal basados en análisis del discurso. Para Foucault (1969) los discursos configuran sistemáticamente los objetos de los que habla, por lo que para Íñiguez (2003) el análisis del discurso permite desenmascarar e identificar 
otras prácticas discursivas, a la vez que se articula como una forma para transformarlas. Esto se basa en el construccionismo social como paradigma, el cual asume que el conocimiento no estaría en la mente de los individuos, siendo más bien un producto del intercambio social (Sisto, 2004). Desde esta perspectiva, el lenguaje actuaría como un constructor de realidad, lo que ayudaría a entender por qué el discurso escrito u oral de los estudiantes sería en sí mismo el significado de sus aprendizajes.

Entre los años 2005 y 2010 se realizó un proceso de sistematización de los aprendizajes identificados por los estudiantes, con el fin de retroalimentar este modelo de formación a través de tres investigaciones, las que fueron contrastadas entre sí para la elaboración de este artículo:

- Año 2005: Tesis de Magíster en Educación, María Julia Baltar.

- Año 2007: Estudio cualitativo de los aprendizajes en la etapa de formación inicial en el área de la Psicología Educacional a través de experiencias de trabajo en terreno.

- Año 2010: Ejercicio de autoevaluación de las asignaturas.

\subsection{Participantes y técnicas de producción de información}

Para el primer estudio se recopilaron en total veinte informes de metaanálisis con base en diarios de aprendizaje, los que como se indicó anteriormente consisten en escritos individuales de análisis metacognitivo desarrollados por todos los estudiantes de las asignaturas. En el segundo estudio se realizaron entrevistas individuales y grupos focales, seleccionando a los estudiantes a través de un muestreo por voluntarios (Salamanca y Martín-Crespo, 2007). Estos alumnos debían cumplir con dos características: haber aprobado las asignaturas y haber cursado todas las asignaturas con la misma docente. En el caso de los grupos focales, estos debían haberse mantenido como grupo de trabajo, al menos, dos semestres académicos. El tercer estudio contó con un análisis de veinte informes de metaanálisis con base en los diarios de aprendizaje.

Siguiendo a Prieto (2001) la recopilación de los metaanálisis consistió en una técnica documental, la que es de relevancia sobre 
todo en los inicios de las investigaciones. Esto lleva a que el segundo estudio buscase complementar los primeros hallazgos con técnicas narrativas, destacando la entrevista focalizada en torno a temas precisos (Alonso, 1998) y dos grupos focales, considerando las recomendaciones de Canales (2006), para volver en el tercer estudio a contrastar los significados con las técnicas documentales utilizadas en el primer estudio. Los metaanálisis tienen un formato que les solicita a los estudiantes dar cuenta, con el máximo de claridad, coherencia y profundidad posibles, de:

- Los aprendizajes y resistencias vividos en relación con las actividades de la asignatura.

- Las condiciones bajo las cuales se desarrollaron los aprendizajes (a través de qué actividades, instancias o acciones, dentro o fuera de las clases).

- Los temas implícitos y factores asociados a los puntos de resistencia.

- Las explicaciones que el estudiante elabora, tanto para los aprendizajes como para las resistencias.

Este ejercicio debiera facilitar la aparición de algunas relaciones entre los temas de otras áreas o disciplinas, lo personal y subjetivo (cognitivo y emocional) y las vivencias analizadas (enlaces con conocimientos, nociones y vivencias previas). Cada estudiante debiera analizar, además, los procesos de atribución en relación con lo vivido, el papel de las relaciones entre teoría y práctica en los aprendizajes y las relaciones que puedan establecer entre las vivencias registradas y analizadas y los procesos de formación como psicólogos/as.

Los criterios de evaluación y calificación incluyen aspectos tales como la claridad, pertinencia, suficiencia, significatividad, diversidad y adecuación de respaldos del metaanálisis, así como relaciones teóricoprácticas, nivel de profundidad en el análisis interpretativo, amplitud en la reflexión crítica, procesos de atribución en torno a lo analizado, fundamentación en acciones decididas, pertinencia en acciones decididas y el grado de conciencia respecto de los aprendizajes y resistencias, todo ello como parte de los procesos reflexivo-críticos respecto de lo analizado en el diario de aprendizajes. 

PSICÓLOGOS/AS, EN DOS UNIVERSIDADES DE LA QUINTA REGIÓN - M. J. Baltar, C. Carrasco, M. Bórquez, C. Cuneo, C. De la Cerda

A continuación se presenta una tabla que resume las características del procedimiento de recopilación de información, así como también de los actores clave en cada estudio considerado para este artículo.

Tabla $n^{0}$ 2. Características del procedimiento de recopilación de información

\begin{tabular}{|l|l|l|}
\hline ESTUDIO 1: AÑO 2005 & $\begin{array}{l}\text { Universidad l } \\
\text { (Tradicional privada) }\end{array}$ & $\begin{array}{l}\text { Universidad 2 } \\
\text { (Privada) }\end{array}$ \\
\hline Tipo de muestreo & Teórico & Teórico \\
\hline $\begin{array}{l}\text { Técnica de producción de } \\
\text { información }\end{array}$ & Documental & Documental \\
\hline $\begin{array}{l}\text { Cantidad de informes de } \\
\text { metaanálisis }\end{array}$ & Seis informes & Cuatro informes \\
\hline $\begin{array}{l}\text { Requisitos para formar parte del } \\
\text { grupo de estudio }\end{array}$ & $\begin{array}{l}\text { Haber cursado y aprobado Taller } \\
\text { de Psicología Educacional II el } \\
\text { año 2004 }\end{array}$ & $\begin{array}{l}\text { Haber cursado y aprobado } \\
\text { Psicología Educacional II } \\
\text { El año 2004 }\end{array}$ \\
\hline Cohorte de los estudiantes & Egresados de la carrera & Cursando 4a año \\
\hline
\end{tabular}

\begin{tabular}{|c|c|c|}
\hline \multicolumn{3}{|l|}{ ESTUDIO 2: AÑO 2007} \\
\hline & $\begin{array}{l}\text { Universidad } 1 \\
\text { (Tradicional privada) }\end{array}$ & $\begin{array}{l}\text { Universidad } 2 \\
\text { (Privada) }\end{array}$ \\
\hline Tipo de muestreo & $\begin{array}{l}\text { Teórico (entrevistas) } \\
\text { Muestreo de casos homogéneos } \\
\text { (Quintana, 2006) / Grupo focal }\end{array}$ & $\begin{array}{l}\text { Teórico (entrevistas) } \\
\text { Muestreo de casos homogéneos } \\
\text { (Quintana, 2006) / Grupo focal }\end{array}$ \\
\hline $\begin{array}{l}\text { Técnica de producción de } \\
\text { información }\end{array}$ & $\begin{array}{l}\text { Dos entrevistas individuales } \\
\text { Un grupo focal }\end{array}$ & $\begin{array}{l}\text { Cinco entrevistas individuales } \\
\text { Un grupo focal }\end{array}$ \\
\hline Número de participantes & 7 & 10 \\
\hline $\begin{array}{l}\text { Requisitos para formar parte del } \\
\text { grupo de estudio }\end{array}$ & $\begin{array}{l}\text { Haber cursado y aprobado Taller } \\
\text { de Psicología Educacional II el } \\
\text { año } 2006\end{array}$ & $\begin{array}{l}\text { Haber cursado y aprobado } \\
\text { Psicología Educacional Il el año } \\
2006\end{array}$ \\
\hline Cohorte de los estudiantes & Egresados de la carrera & Cursando $4^{\circ}$ año \\
\hline
\end{tabular}

\begin{tabular}{|l|l|l|}
\hline ESTUDIO 3: AÑO 2010 & $\begin{array}{l}\text { Universidad 1 (Tradicional } \\
\text { privada) }\end{array}$ & $\begin{array}{l}\text { Universidad 2 } \\
\text { (Privada) }\end{array}$ \\
\hline Tipo de muestreo & Teórico & Teórico \\
\hline $\begin{array}{l}\text { Técnica de producción de } \\
\text { información }\end{array}$ & Documental & Documental \\
\hline $\begin{array}{l}\text { Cantidad de informes de } \\
\text { metaanálisis }\end{array}$ & Cinco informes & Cinco informes \\
\hline $\begin{array}{l}\text { Requisitos para formar parte del } \\
\text { grupo de estudio }\end{array}$ & $\begin{array}{l}\text { Haber cursado y aprobado Taller } \\
\text { de Psicología Educacional II el } \\
\text { año 2009 }\end{array}$ & $\begin{array}{l}\text { Haber cursado y aprobado } \\
\text { Psicología Educacional II } \\
\text { El año 2009 }\end{array}$ \\
\hline Cohorte de los estudiantes & Egresados de la carrera & Cursando 4ºño \\
\hline
\end{tabular}




\subsection{Procedimiento de análisis}

Se llevó a cabo un análisis crítico del discurso siguiendo las recomendaciones de Parker (1996) a través del siguiente procedimiento: aludir a las formas de habla como objetos de estudio, suscitar una red de relaciones alrededor de los objetos que alude el texto, develar las reglas culturales encubiertas e identificar los contrastes entre las distintas formas de habla.

Es así como finalmente lo que se presenta en este artículo es un análisis de los significados de los estudiantes respecto de su proceso de aprendizaje en el marco de la formación recibida bajo la modalidad de talleres educativos, recurriendo a los discursos textuales para su descripción y problematización.

Hemos optado por presentar este análisis no de forma categorial, sino más bien organizado según la evolución que habrían vivido, ya que se trata del análisis de una experiencia situada en permanente reformulación. De esta manera, hemos querido respetar el carácter de construcción y revisión permanente de la propuesta de innovación pedagógica en el contexto de la educación superior, profundizando las implicancias teóricas en las discusiones de este artículo.

A continuación se detalla la metodología de los tres estudios:

Tabla $\mathrm{n}^{0}$ 3. Procedimiento de los procesos de investigación.

\begin{tabular}{|l|l|l|l|}
\hline & 2005 & 2007 & 2010 \\
\hline Paradigma & $\begin{array}{l}\text { Sociocrítico } \\
\text { construccionista }\end{array}$ & Sociocrítico construccionista & $\begin{array}{l}\text { Sociocrítico } \\
\text { construccionista }\end{array}$ \\
\hline $\begin{array}{l}\text { Participantes y técnicas de } \\
\text { producción de información }\end{array}$ & Diez metaanálisis & $\begin{array}{l}\text { Siete entrevistas individuales } \\
\text { Dos grupos focales }\end{array}$ & Diez metaanálisis \\
\hline Análisis & $\begin{array}{l}\text { Análisis crítico del } \\
\text { discurso }\end{array}$ & Análisis crítico del discurso & $\begin{array}{l}\text { Análisis crítico } \\
\text { del discurso }\end{array}$ \\
\hline
\end{tabular}

\subsection{Consideraciones éticas}

Resulta especialmente importante especificar las consideraciones éticas de los tres estudios contrastados, debido a que la mayoría de las autoras de este artículo conforma el equipo docente de las cátedras investigadas. Al respecto es importante señalar, en primer lugar, que se firmaron consentimientos informados con el fin de resguardar 
la confidencialidad de la información, tanto de las universidades involucradas como de la identidad de los sujetos que participaron en el estudio.

Respecto del procedimiento de análisis de los informes de metaanálisis, estos consentimientos informados consideraron la autorización expresa de los estudiantes autores de estos documentos. Por su lado, las entrevistas individuales y los grupos focales se realizaron de forma cruzada por quienes estaban trabajando por entonces como ayudantes de ambas cátedras. En este sentido, quien era ayudante en la universidad 1 realizó las entrevistas a los estudiantes de la universidad 2 y quien era ayudante de la universidad 2 realizó las entrevistas a los estudiantes de la universidad 1. De igual modo, tanto las entrevistas como los grupos focales contaron con la presencia de una ayudante de investigación que forma parte de la autoría de este artículo y que no ha participado en el equipo docente en ninguna de las dos universidades.

\section{Resultados}

\subsection{El inicio de los discursos}

En el año 2005 la construcción del conocimiento por parte de los estudiantes posee un carácter subjetivo en el discurso, vislumbrando un compromiso afectivo con la vivencia y necesidad de significarla en relación con la propia identidad personal y profesional.

(...) lejos los años que han sido más trascendentales para mí, tanto por un proceso académico, individual y grupal, han sido los dos últimos años de esta carrera. (Extracto metaanálisis).

Aparece así un sentido de trascendencia de los aprendizajes, ya que los estudiantes manifiestan que algunos de sus aprendizajes han impactado en otros ámbitos de su vida, dando cuenta de un posicionamiento frente al conocimiento y la acción profesional vinculados a la posibilidad de interactuar con otros. En esta misma lógica, los estudiantes relegan la obtención de calificaciones a un lugar secundario en su formación, valorando la construcción de 
conocimientos significativos como un elemento central en sus procesos formativos, por sobre el resultado objetivable de los mismos.

En este sentido, creo haber perdido hace bastante tiempo el compromiso por una nota académica, por el contrario, una búsqueda desinteresada por relacionarme con los actores educativos para la construcción de una realidad educacional alternativa. (Extracto metaanálisis).

En la validación y reconocimiento del proceso por sobre el resultado, destaca la significación de los fines del trabajo llevado a cabo en las cátedras, destacando la noción de estar colaborando con una educación alternativa a la tradicional.

Por otra parte, algunos estudiantes si bien se dan cuenta de la importancia de las nuevas significaciones construidas, identifican como relevante la fuerza de los marcos de referencia personales y conocimientos previos que influyen en sus dificultades para continuar sus procesos de aprendizaje. En este sentido, relevan una concepción de aprendizaje que se caracteriza por la recursividad, siendo una de sus manifestaciones la presencia de contradicciones:

Así como hemos disfrutado de nuevas significaciones, también es necesario experimentar retrocesos que nos lleven cada vez a un cuestionamiento mayor de nuestras propias prácticas y aunque desde un pensamiento más crítico podamos modificar algunas conductas, nuestros marcos de referencia son aún muy potentes y nos llevan a caer en contradicciones. (Extracto metaanálisis).

El supuesto implícito que aparece en estos discursos es la idea de que los cambios conductuales resultan más sencillos de evidenciar que los cambios a nivel de sentidos, pensamientos o creencias, siendo evaluados estos últimos como cambios más profundos e, incluso, más duraderos en el tiempo.

Vinculado con lo anterior algunos estudiantes dan cuenta de movimientos en su mirada respecto de su historia personal y estudiantil, manifestando haberse dado cuenta de su postura fatalista y carente de sueños, en un intento por comprenderse a sí mismos y 
a los sujetos con los cuales interactuaban en los trabajos de campo. Pese a esto, algunos estudiantes visualizan una salida del fatalismo que han experimentado al manifestar el reconocimiento de que es posible cambiar la realidad. Desde esta perspectiva, el cambio social pasaría por pequeños cambios en las relaciones sociales que fueron construyendo en los trabajos de campo, a partir de lo cual manifiestan haber reconstruido antiguos sueños.

(...) porque ni siquiera sabía cuáles eran y dónde estaban mis sueños. (...) creo que poco a poco he podido ir construyendo (o reencontrándome) con mi sueño, que tiene ribetes contraculturales y que se relaciona con la sociedad que quiero construir: un todo de sentido. (...) nos dieron la oportunidad de soñar y de aprender a establecer relaciones contraculturales, convenciéndonos de que es posible. Desde ahí, creo que el cambio social es posible, desde estas pequeñas cosas que me han marcado no solo en mi formación (...) (Extracto metaanálisis).

De esta manera, se deja entrever una tensión en las formas de concebir la realidad social: por un lado se asume que la realidad es superior a los sujetos que la construyen, o bien, que forman parte de ella, visualizándose concepciones de tipo estructuralistas, las que estarían en la base de las sensaciones de fatalismo declaradas por los estudiantes. Por otro lado, aparece una especie de resignificación de la noción de realidad, como si esta fuese localmente construida. Sin embargo, no queda claro si esta segunda mirada de la realidad operaría como una forma de recuperar la esperanza perdida frente al fatalismo del determinismo estructural o si, efectivamente, estaría dando cuenta de una tensión paradigmática en las nociones de realidad y cambio de los estudiantes.

Los informes de metaanálisis analizados en este momento tenderían a focalizarse mayoritariamente en la vivencia profesional, es decir, se encontrarían centrados más bien en el vínculo estudiantetrabajo en terreno, por sobre los componentes de la propia historia de vida involucrados en los procesos de aprendizaje. 


\subsection{Avanzando en el camino}

En el año 2007, los estudiantes de las entrevistas comienzan a hacer referencia a sus propias historias de vida. Algunos hacen alusión a una historia escolar previa a su ingreso a la universidad, la que condicionaría ciertos procesos educativos que viven al interior de su formación como psicólogos y psicólogas. Por un lado, esta historia previa predispondría a los estudiantes a rechazar inicialmente las temáticas vinculadas con la educación, trasladando este rechazo hacia el área de psicología educacional.

\section{(...) uno como que de chico como que te van reprimiendo esa habilidad, por lo menos en ese tipo de colegios, y llegai acá que tú tenís que ser crítico, esa es como la idea, y te cuesta como desarrollar esa parte. (Extracto entrevista individual).}

Es así como algunos estudiantes ubican su dificultad de análisis crítico en la historia educativa, restando importancia a su propia responsabilidad en este proceso. Bajo esta visión, los estudiantes ubicarían el cambio fuera de ellos, desarrollando procesos de atribución externa. Sin embargo, algunos estudiantes hacen alusión a una habilidad más bien "reprimida" desde el sistema educativo, dando cuenta de que la posibilidad de cuestionamiento crítico no habría sido promovida por el sistema escolar, por lo que encontraban en estas cátedras un espacio para expresarla.

(...) en ese tiempo me sentía como súper excluido, pero como que volvió todo eso de haberme sentido así casi como el malo de haber criticado (...) entonces como que casi me sentí completo y feliz, un buda, no sé, pero fue como claro, haber traído toda esa energía que fue como reprimida en mis años escolares y haberla ocupado, es como un reencontrarse con uno mismo. (Extracto entrevista individual).

A partir de este punto es posible analizar cómo el ser humano es visualizado en tensión entre una concepción pasiva y activa del mismo, enfatizando el papel del entorno. Por un lado, el sistema escolar sería significado como el responsable de una aparente mala 
actitud inicial a tocar los temas educativos en el contexto de su formación como psicólogos, a la vez que se perciben a sí mismos como los responsables de superar ese desdén inicial. En este sentido, para algunos estudiantes las asignaturas del área de psicología educacional constituyen un espacio posibilitador para la emergencia del potencial transformador de sí mismos y de sus entornos.

(...) te estaban retando, pero en el fondo igual te estaban apoyando, o sea a mí me quedaba claro que las cosas que nos decían no eran para cagarnos (sic), sino que era: "oye, miren, vean, vean", eso yo creo que era fundamental. (Extracto entrevista individual).

Sin embargo, para algunos estudiantes este espacio se presenta muy lejano de sus propias maneras de construir relaciones al interior de la educación, manifestando un deseo por asimilar acríticamente la propuesta de la asignatura, solo porque se encuentra presente en la malla curricular.

Yo creo que otro mito que hay con (psicología) educacional, es que cuando entrai a su ramo, tenís que entrar a bailar su vals. (Extracto grupo focal).

A partir de esta visión es posible plantear que el carácter explícitamente crítico de las asignaturas se presentaría a sí mismo como una gran paradoja, ya que tendría un potencial discriminador, jerarquizando unos conocimientos por sobre otros en donde estos (los conocimientos) son percibidos al comienzo de las asignaturas como un acto de imposición. Sin embargo, para algunos estudiantes la posición facilitadora de la docente y su equipo permite paulatinamente ir avanzando en una comprensión más cercana.

(...) sentir un espacio así como súper cuidadoso, al menos yo lo sentía así, ustedes facilitaron que el espacio fuera como súper cuidadoso pa' que todos pudieran hablar sin sentirse amenazados ni criticados ni nada y eso pa' mí tenía como harto sentido. (Extracto entrevista individual). 
Los estudiantes que consideran que comparten la responsabilidad en el proceso de enseñanza y aprendizaje incluyen la temática del poder en sus reflexiones, relevando la historia de la psicología como disciplina que se ha ubicado desde la experticia, no solo en su relación con la educación, sino en su relación con el conocimiento, transfiriendo los aprendizajes de estas asignaturas al conocimiento disciplinar.

Es todo un tema del poder, cachai, el poder de, de que tenís que, que el poder que tiene el psicólogo, es como todo el juego, el juego del poder, cachai, lo tenís que ver. (Extracto entrevista individual).

(...) este ramo me hizo acercarme al ser psicólogo y reencantarme con la carrera, reencantarme con ser más activo dentro de la Escuela, tomar otros roles dentro de mi misma educación, en el fondo fue casi un punto crítico en mi educación (...) de no haber sido nunca el protagonista de nada, me hizo cambiar mucho. (Extracto entrevista individual).

\subsection{Cómo estamos hoy}

En los análisis que desarrollan los estudiantes durante el año 2010 aparece un inicio de frustración, ya que las dificultades son evaluadas cada vez como más entorpecedoras del cumplimiento de los objetivos que estos estudiantes traían al momento de ingresar a cada colegio en el que debían llevar a cabo el trabajo en terreno.

La búsqueda del colegio es algo para lo cual no todos estamos preparados, la frustración es solo uno más de los tantos ingredientes que conforman este pastel que se llama educación. (Extracto metaanálisis).

La desesperanza que se evidencia en el discurso anterior estaría mediatizada por un proceso de atribución externa de responsabilidad, en cuanto se asumiría que es el sistema educativo el que estaría dificultando el ingreso y desarrollo de las actividades planificadas por los estudiantes, dejando de lado la propia responsabilidad frente a las decisiones profesionales y sus consecuencias. 

PSICÓLOGOS/AS, EN DOS UNIVERSIDADES DE LA QUINTA REGIÓN - M. J. Baltar, C. Carrasco, M. Bórquez, C. Cuneo, C. De la Cerda

Ese cambio que nos alentó y energizó en un comienzo chocó violentamente con un sistema educativo que se encargaba de torpedear nuestras ganas de trabajar constantemente. (Extracto metaanálisis).

Sin embargo, frente a esta desesperanza, algunos estudiantes plantean una necesidad de cuestionarse acerca de la noción del cambio educativo y el contexto en el que este debiera surgir. En este sentido se valora la necesidad de analizar críticamente el sentido del propio cambio con los docentes con los que se trabaja en las actividades en terreno.

Lo mismo con los profesores, si se planteaba una posibilidad de cambio, cualquiera fuera, sin tener siquiera la posibilidad de cuestionársela, debatirla, pensársela, recibirla críticamente, posiblemente cualquier intervención pase sin pena ni gloria por el establecimiento. (Extracto metaanálisis).

Ante la pregunta ies posible el cambio en educación? Lo primero que se me viene a la mente es cuestionarme qué entendemos por cambio, qué entiendo yo por cambio. (Extracto metaanálisis).

En este contexto de cambio educativo, los estudiantes comienzan a cuestionar su propio papel como psicólogos, analizando la responsabilidad que les compete como agentes transformadores, concluyendo que el cambio debe comenzar desde los propios sujetos de cambio. Este aprendizaje se vuelve relevante, puesto que lo que implícitamente ocurre es el cuestionamiento del poder de la psicología y de su papel en los procesos de aprendizaje de los grupos y las organizaciones: es el tema del poder y de cómo se ejerce lo que se estaría poniendo en evidencia.

(...) considero que como futuros psicólogos(as) solo podemos actuar como facilitadores sociales, puesto que ni siquiera podemos incentivar el cambio, ya que a mi parecer, según mi propia experiencia en el liceo, el verdadero cambio solo puede surgir desde la misma organización. (Extracto metaanálisis). 
Es así como el papel de la psicología y de los psicólogos, específicamente, aparece otra vez cuestionado, destacando un aprendizaje que enfatiza la necesidad de construir una identidad profesional centrada en la facilitación y comprensión empática de los individuos.

La pregunta es válida entonces, ¿cuál es el papel de los psicólogos en este baile venido a menos? Es una pregunta que me he hecho durante todo este semestre (...) Lo primero que viene a mi mente es "facilitar" (...) por sobre todo comprensión empática, de hecho esta última es aquella que más sentido me hizo, por cuanto comprender empáticamente significa adentrarse en un proceso que busca un contacto real con otro, que es diferente, más allá de juzgarlo o evaluarlo, que es finalmente a lo que profesores, directivos y alumnos están sometidos de forma majadera y por lo mismo hastiados. (Extracto metaanálisis)

Sin embargo, hay estudiantes que plantean que desde esta posición muchas veces ocurriría un enmascaramiento de la disciplina y de sus intereses, llevando a los psicólogos a creer que la comprensión empática otorga el poder de hablar por otros, destacando nuevamente el papel de las relaciones de poder en las intervenciones de la disciplina. Con esto, ocurre una transferencia desde la disciplina vinculada a la educación hacia otras áreas de desempeño profesional relacionadas con los grupos y las organizaciones.

Desde mi propia experiencia por lo realizado en este taller educacional y en otros talleres como el taller organizacional (comunitario) donde también comenzamos intentando comprender la realidad de un colectivo, para luego desde esta comprensión ir detectando "nodos de conflicto", con el fin de posteriormente coconstruir con los propios implicados espacios donde se pueda reflexionar, abrir nuevas miradas y ver con ellos qué tanto sentido le hacen nuestras apreciaciones de su realidad y a qué conclusiones llegan ellos mismos, me doy cuenta en la práctica que a pesar de que uno se intente posicionar desde la psicología como un facilitador social puede caer 
en la trampa de creer estar comprendiendo la realidad del otro, pero al final puede estar ignorando o, incluso, atentando contra la cultura imperante, en este caso de la escuela. (Extracto metaanálisis).

Finalmente, los estudiantes dan cuenta de cómo el tránsito de las asignaturas los ha llevado a cuestionarse acerca del propio papel como interventores socioeducativos. En efecto, asumen que este es el tránsito de una identidad de alumno hacia una profesional donde los conocimientos académicos cobran un lugar secundario, destacando la capacidad de analizar críticamente la realidad, permitiendo entonces promover procesos de problematización en los espacios sociales en los que trabajarán en el futuro y apostando por un cambio en pequeñas dimensiones.

(...) al momento de comenzar a trabajar en establecimientos educacionales, en cuarto (año), lo hicimos desde la postura con la que enfrentábamos todos los trabajos prácticos, desde nuestro rol de estudiantes, (...) sin embargo en el camino nos dimos cuenta de que esto no era lo más importante, sino que de alguna manera lo importante radicaba en la mirada, (...) para poder distinguir en dónde es posible intervenir, apoyar, fomentar, problematizar, aportar una perspectiva distinta... y ahí vimos cómo los cambios parten también de cosas aparentemente pequeñas (...). (Extracto metaanálisis).

\section{Discusiones}

Para estos estudiantes el cambio muchas veces es ubicado como proveniente desde afuera, promoviendo así procesos de atribución externa (López y otros, 1991) y haciéndose cómplices del sistema social que, en su discurso, los oprimiría. Sin embargo, al hacer alusión a una habilidad más bien "reprimida" desde el sistema educativo, algunos estudiantes estarían abriendo un nuevo foco de análisis desde el cual quedaría en evidencia cómo la posibilidad de cuestionamiento crítico en realidad siempre estuvo presente, al menos como promesa incumplida. 
En este caso, la concepción de ser humano en la base del discurso de estos estudiantes se encontraría influenciada por la posibilidad de transformación como potencial, aproximándose a la noción de agenciamiento humano (Giroux, 1983) antes mencionada. En este sentido, para algunos estudiantes es el espacio de las asignaturas del área de psicología educacional, un espacio posibilitador para la emergencia del potencial transformador de sí mismos y de sus entornos.

Sin embargo, en algunos estudiantes se ven dificultados sus procesos de agenciamiento, aludiendo a que este espacio social se presenta de forma contracultural y poco contextualizada respecto de sus propias maneras de construir relaciones al interior de la educación. Así es posible plantear que, en términos de Bourdieu y Passeron (2002) las asignaturas del área se ubicarían en la vivencia de los estudiantes como un campo de producción cultural con ciertas características de élite, ya que se encuentran enmarcadas en el contexto de la educación superior. De esta manera, para muchos estudiantes, los capitales más relevantes en este campo social son los culturales, por lo que buscarían apropiarse y acumular la mayor cantidad de este tipo de capital para actuar como protagonistas de este campo social. A partir de lo anterior sería posible comprender el deseo de algunos estudiantes por asimilar acríticamente la propuesta de las asignaturas solo porque se encuentra presente en la malla curricular, asumiendo que para conseguir el título profesional sería necesario adscribirse a la propuesta pedagógica de las asignaturas del área. En este afán por pertenecer a este campo de producción cultural se corre el riesgo de rechazar los capitales culturales de ingreso, es decir, los capitales culturales de los campos de producción cultural de proveniencia de los propios estudiantes. Si el proceso se vuelve lineal y cada estudiante enfatiza la asimilación de los capitales de este campo de forma acrítica, se corre el riesgo de caer en confusiones que no permitan comprender el sentido de la propuesta de las asignaturas, instrumentalizando el discurso propuesto o, simplemente, sin entender de qué se trata.

Frente a este fenómeno en el discurso de algunos estudiantes, la docente y sus ayudantes facilitarían, como parte del mismo campo social, un espacio de análisis, reflexión, autocuestionamiento y 
acompañamiento de lo que puede llegar a implicar la participación en un nuevo campo de producción cultural. De alguna manera, entonces, sería posible plantear que este proceso en términos iniciales, al menos, se relacionaría con una práctica de violencia simbólica (Bourdieu y Passeron, 2002). Para ello, el efecto de la acción pedagógica sería siempre simbólico y respondería a los intereses y objetivos de la universidad y su función como promotora social.

Sin embargo, sería posible plantear que el carácter explícitamente crítico de las asignaturas se presentaría a sí mismo como una gran paradoja en cuanto poseería un potencial reproductivo, al jerarquizar y discriminar conocimientos. Estos, a su vez, son percibidos por parte de los estudiantes como naturalmente jerarquizados (al menos al comienzo de las asignaturas) y asumen la propuesta de la cátedra como una imposición a la cual le buscan un sentido adaptativo y mostrando, finalmente, la efectividad de una violencia simbólica ejercida por parte de la docente y de su equipo de ayudantes. Sin embargo, para algunos estudiantes esta posición facilitadora permite la comprensión de los capitales culturales más relevantes para conocer y comprender, al menos, cómo funciona este campo de producción cultural. El ejercicio de violencia simbólica, no obstante, seguiría presente en cuanto la autoridad pedagógica se encuentre reconocida como una autoridad legítima, disimulando el poder arbitrario, lo que ocurre cuando los estudiantes reconocen la legitimidad de una instancia pedagógica, pero no comprenden el funcionamiento de la relación de fuerzas en que están situados. Este es el punto en el cual la autoridad pasa a ser considerada autoritarismo e imposición, presentando una tensión que busca ser resuelta cuando se analiza la posición de la docente como una figura de contención.

Pese a lo anterior, un trabajo de develamiento de las fuerzas ocultas en el ejercicio pedagógico de estas asignaturas podría llevar a la superación de una relación de violencia simbólica, permitiendo entonces la transformación de la misma y de sus condiciones de producción. Es posible pensar que este trabajo se lleva a cabo durante el avance de las asignaturas, donde los estudiantes vivencian ciertos quiebres que darían cuenta de un proceso de reflexión y develamiento de la propia acción y de la acción educativa de la que formarían parte. 
De todos modos, este ejercicio de violencia simbólica no ocurriría tan solo al interior de estas asignaturas, sino que se trataría de una característica del espacio educativo de la universidad, ya que al ser este un espacio con bastantes características de élite, la violencia es deseada y compartida, volviendo a los estudiantes cómplices de la misma.

Sin embargo, los estudiantes que consideran que comparten responsabilidad en el proceso de enseñanza y aprendizaje incluyen la temática del poder en sus reflexiones, relevando la historia de la psicología como disciplina que se ha ubicado a sí misma desde la experticia, no solo en su relación con la educación, sino en todas las relaciones que tradicionalmente ha establecido con el conocimiento, refiriendo a las dimensiones éticas y políticas (Montero, 2001) de la psicología, así como también a la posición del psicólogo y su vinculación con el poder (Wiesenfeld, 2000). De esta manera, algunos estudiantes logran visualizar en sí mismos la posibilidad de mantención o transformación del statu quo.

Sin embargo, estos estudiantes mantienen una visión individualizada del cambio social, centrándose más bien en el ser psicólogo individual que se vuelca sobre sí, por sobre la relevancia del colectivo. De todos modos, existirían contradicciones al interior de este discurso, posicionando a algunos estudiantes como agentes de cambio social.

Es así como el cambio posible queda en las manos de los propios estudiantes de psicología, los que debieran reconocer el papel de las estructuras sociales para desde allí generar espacios y prácticas que cuestionen la psicología tradicional y proponer una redefinición de la misma. En este análisis, las cátedras de psicología educacional se sitúan como espacio posibilitador para esta transformación.

Finalmente, es posible concluir que estos aprendizajes serían identitarios, es decir, construirían un psicólogo/a consciente de las implicancias éticas y sociales de su propio rol, transfiriendo los aprendizajes de estas cátedras a otras áreas de la disciplina. Lo anterior se puede ver graficado en la siguiente figura: 

PSICÓLOGOS/AS, EN DOS UNIVERSIDADES DE LA QUINTA REGIÓN - M. J. Baltar, C. Carrasco, M. Bórquez, C. Cuneo, C. De la Cerda

Figura $\mathrm{n}^{\circ} 1$. Aprendizajes comunes en estudiantes de psicología

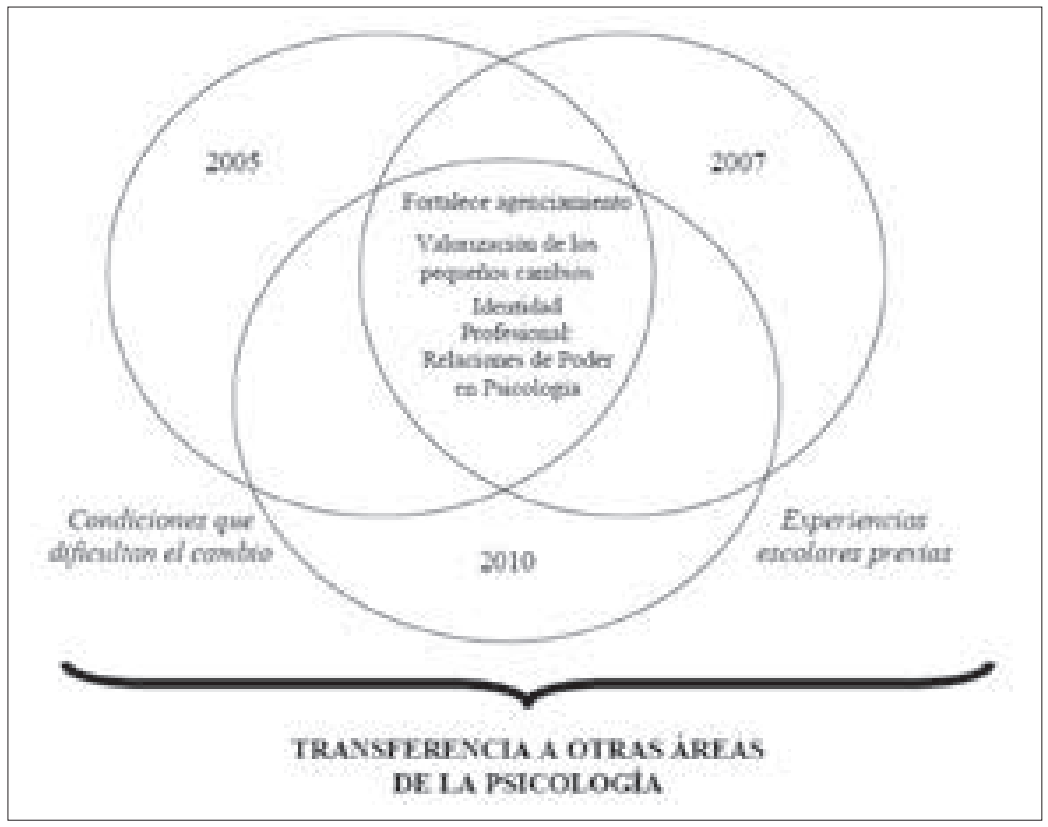

En esta figura vemos cómo las condiciones que dificultan el cambio estarían dadas por elementos que provienen, esencialmente, desde el contexto en el que se desarrollan las cátedras, más que desde las cátedras y su propuesta metodológica mismas, destacando sobre todo el hecho de que estas experiencias de enseñanza y aprendizaje se encuentran enmarcadas en el papel y función social de la universidad como institución social de élite. Respecto de esto mismo la escuela de origen de los estudiantes (como otra institución social) sería representada en las experiencias previas como reproductora de discursos y prácticas altamente alienantes, que los convierten en "sujetos de resultados". Para estos estudiantes lo anterior significa que la experiencia escolar previa aparece como una vivencia que los empuja a creer que la educación está hecha para reproducir acríticamente los contenidos, con el único fin de obtener buenos resultados (expresados en el rendimiento académico).

Estas dos condiciones, que dificultan el cambio y están vinculadas con la función social de la universidad y las experiencias 
escolares previas, se quiebran en el espacio de las cátedras, generando tensiones que no siempre son resueltas. Sin embargo, cuando estas logran superarse, se desarrolla una conciencia crítica respecto del propio entorno, que lleva a los estudiantes a agenciarse en la medida en que logran resignificar su identidad profesional, construyendo discursos en los que ellos se posicionan como encargados de llevar a cabo transformaciones micropolíticas que cuestionan y buscan transformar las relaciones de poder que establece la psicología como disciplina cuando interviene en el ámbito escolar.

Estos resultados nos hacen pensar que un desafío pendiente en la metodología de los talleres educativos como experiencia de formación de psicólogos educacionales se encuentra en construir e incluir estrategias que permitan a todos los estudiantes resolver la tensión generada entre las condiciones que dificultan el cambio de concepción y la propuesta de la asignatura, permitiendo entonces un verdadero espacio de resignificación de la propuesta que aparece en un primer momento como un acto de violencia simbólica. Además, resultaría interesante construir una línea de investigación que permitiera, por un lado, comprender de mejor forma el papel del contexto universitario como promotor, o bien, obstaculizador de cambios en la identidad de los psicólogos y, por otro, que permita comprender con mayor profundidad y claridad los componentes de la identidad profesional que entran en crisis (y son resignificados) a la luz de esta propuesta.

\section{Referencias bibliográficas}

Alonso, L. (1998). La mirada cualitativa en sociología. Madrid: Fundamentos.

ANPSE (2011). Página Web Asociación Nacional de Psicólogos Educacionales del Colegio de Psicólogos de Chile http://www.colegiopsicologos.cl/elcolegio/asociaciones/asociacion-nacional-de-psicologos-educacionales/

Avendaño, C. (1996). Formación profesional del psicólogo. Antecedentes acerca de la formación del psicólogo en Chile. Terapia Psicológica, 26, VI (2), pp43-47.

Baltar, M. (2005). Estudio cualitativo de los aprendizajes en la etapa de formación inicial en el área de la Psicología Educacional a través de experiencias de trabajos en terreno; una mirada desde el discurso de los estudiantes de 
158 EL TALLER EDUCATIVO: SISTEMATIZACIÓN DE UN MODELO DE FORMACIÓN DE PSICÓLOGOS/AS, EN DOS UNIVERSIDADES DE LA QUINTA REGIÓN - M. J. Baltar, C. Carrasco, M. Bórquez, C. Cuneo, C. De la Cerda

dos instituciones formadoras de psicólogos. Tesis para optar al Grado de Magíster en Educación Mención Currículo, Pontificia Universidad Católica de Valparaíso. Viña del Mar, Chile.

Bernasconi, A. y Rojas, F. (2003). Informe sobre la Educación Superior en Chile: 1980-2003. Disponible en http://unesdoc.unesco.org/ images/0014/001403/140395s.pdf

Bourdieu, P. y Passeron, J. (2002). La reproducción: elementos para una teoría del sistema de enseñanza. Barcelona: Laia.

Canales, M. (2006). Metodologías de la investigación social: Introducción a los oficios. Santiago de Chile: LOM.

Consejo Nacional de Educación (2011) Bases Índices. Disponible en http:// www.cned.cl/public/Secciones/SeccionIndicesEstadisticas/indices_ estadisticas_BDS.aspx

Comité Coordinador de sicólogos/as del MERCOSUR (2001) Documento referido a la formación del psicólogo en los países del Mercosur y países asociados. Comité Coordinador de psicólogos/as del MERCOSUR.

Coll. C., Marchesi, A. y Palacios, J. (1990). Desarrollo psicológico y Educación. Tomo II Psicología de la Educación. Madrid: Alianza Editorial.

Cerda, A., Aránguiz, G., Cid, S. y Miranda, H. (1994). Los docentes y los procesos de descentralización pedagógica. Santiago de Chile: Instituto de Perfeccionamiento Colegio de Profesores y PIIE.

Del Mazo, G. (1966). El movimiento de la reforma universitaria en América Latina: sintesis explicatoria. Lima: Universidad Nacional Federico Villareal.

Descouvriers, C. (1999). Lo psicológico en los anales de la Universidad de Chile. Santiago: Impresos Universitarios.

Foucault, M. (1969). La arqueología del saber. México: Siglo XXI.

Freire, P. (2003). El grito manso. Buenos Aires: Siglo Veintiuno Editores.

Giroux, H. (1983). Teorías de la reproducción y de la resistencia en la nueva sociología de la educación: un análisis crítico. Harvard Educational Review 3 pp. 257-294.

Hernández Arista, J. (2000). La supervisión. Un sistema de asesoramiento y orientación para la formación y el trabajo. Valencia: Nau Llibres.

Iñiguez, L. (2003). El lenguaje en las ciencias sociales: fundamentos, conceptos y modelos. En Iñiguez, L (2003) (Ed.) Análisis del discurso. Manual para las ciencias sociales. Pp. 43- 82 Barcelona: Editorial UOC.

López, Assàel y Neumann (1991). La cultura escolar ¿Responsable del fracaso? Santiago de Chile: PIIE. 
López, V., Carrasco, C., Morales, M., Ayala, A., López, J. y Karmy, M. (2011) Individualizando la violencia escolar: análisis de prácticas discursivas en una escuela municipal de la Región de Valparaíso PSYKHE, 20(2) pp. 7-23.

Linn, H. (2007). La situación laboral del psicólogo en Chile. Ponencia para el VII. Congreso Nacional de Psicología 9 y 10 de noviembre 2007, Santiago de Chile.

Makrinov, N., Scharager, J. y Molina, L. (2005). Situación actual de una muestra de psicólogos/as egresados de la Pontificia Universidad Católica de Chile. Psykhe, 14(1) pp. 69-77.

Meller,P., Lara, B. y Valdés, G. (2009). Carreras Universitarias (por región): Comparación interregional de ingresos al primer año y probabilidad de encontrar empleo. Santiago de Chile: Ministerio de Educación. Universidad de Chile

Montero, M. (2001). Ética y política en psicología. Las dimensiones no reconocidas. Disponible en: http://psicologiasocial.uab.es/athenea/index.php/ atheneaDigital/article/view/1

Morales, M., Sziklai, G., Díaz, R. y Scharager, J. (1988). La formación profesional de los psicólogos en Chile: análisis de la opinión de los psicólogos y estudiantes de la carrera. Revista Chilena de Psicología 9(1) pp. 57-64.

Parker, I. (1996). Discurso, cultura y poder en la vida cotidiana. En Gordo, A. y Linaza, J (1996) (Comp.) Psicologías, discursos y poder. Pp. 79-92. Madrid: Visor.

Prieto, M. (2001). La investigación en el aula zuna tarea posible? Valparaíso: Ediciones Universitarias de Valparaíso de la Universidad Católica de Valparaíso.

Quintana, A. (2006). Metodología de investigación científica cualitativa. En Quintana, A. y Montgomery, W. (2006). Psicología: tópicos de actualidad. pp. 47-84 Lima: UNMSM.

Salamanca, A. y Martín-Crespo, C. (2007). El muestreo en la investigación cualitativa. Nure Investigación, 27 p.14.

Sisto, V. (2004). Ideas que se mueven. Los caminos del socioconstruccionismo desde el discursivismo a las Actividades Dialógicas Corporizadas. Revista de Psicología Universidad de Valparaíso. 2(1) pp. 1-22.

Urrutia, C. (2002). La psicología en Chile. Infocop, Consejo General de Colegios Oficiales de Psicólogos no 82. Disponible en http://www.cop.es/infocop/ vernumero.asp?id=1038 
160 EL TALLER EDUCATIVO: SISTEMATIZACIÓN DE UN MODELO DE FORMACIÓN DE PSICÓLOGOS/AS, EN DOS UNIVERSIDADES DE LA QUINTA REGIÓN - M. J. Baltar, C. Carrasco, M. Bórquez, C. Cuneo, C. De la Cerda

Villegas, J. F. (1998). Demandas del desarrollo para la psicología en Chile. Terapia Psicológica, VII (1), pp. 41-51.

Vilanova, A. (2003). La formación académica del psicólogo. En Vilanova, A. Discusión por la psicología. Pp. 175-189. Mar del Plata: Universidad Nacional de Mar del Plata.

Winkler, M., Pasmanik, D., Alvear, K. y Reyes, M. (2007). Cuando el bienestar psicológico está en juego: la dimensión ética en la formación profesional de psicólogos y psicólogas en Chile. Terapia Psicológica, 25 (001) pp. 5-24.

Wiesenfeld, E. (2000). Entre la prescripción y la acción: la brecha entre la teoría y la práctica en las investigaciones cualitativas. Forum qualitative social research (On line Journal), 1 (2). Recuperado en mayo 2007: http://qualitative-research.net/fqs/fqs-e/2-00inhalt-e.htm

Recibido: 03/07/2012

Aceptado: 24/10/2012 